\title{
Investigation on the relationship of dimensions of the maxillary sinus drainage system with the presence of sinusopathies: a cone beam computed tomography study
}

\author{
Ana Beatriz Gomes de Carvalho ${ }^{\mathrm{a}}$, Andre Luiz Ferreira Costa ${ }^{\mathrm{b}, *}$, Acácio Fuziy ${ }^{\mathrm{b}}$, \\ Afonso Celso Souza de Assis ${ }^{\mathrm{a}}$, José Ribamar Castro Veloso ${ }^{\mathrm{b}}$, \\ Luiz Roberto Coutinho Manhães Junior ${ }^{\mathrm{a}}$, Mauro Pedrine Santamaria ${ }^{a}$, \\ Sérgio Lucio Pereira de Castro Lopes ${ }^{\mathrm{a}}$
}

a Department of Diagnosis and Surgery, São José dos Campos School of Dentistry, São Paulo State University (UNESP), São José dos Campos, SP, Brazil
${ }^{\mathbf{b}}$ Department of Orthodontics and Radiology, University of São Paulo City (UNICID), São Paulo, SP, Brazil

\section{A R T I C L E IN F O}

\section{Keywords:}

Diagnostic imaging

Paranasal sinus

Anatomy

\begin{abstract}
A B S T R A C T
Objective: This study sought to assess the relationship between the dimensions of the maxillary sinus drainage system with the content of sinuses.

Design: Three-hundred cone beam computed tomography images were selected from a database (105 males and 195 females). The images were assessed by a single investigator, trained and calibrated, performing image analysis. The length of the infundibulum and the ostium height in both maxillary sinuses were measured. The data were analyzed using Minitab 16 , using $5 \%$ as a critical $p$-value.

Results: A significant gender difference was also found for presence in the normal content of sinus for females and presence of antral pseudocyst for males $(\mathrm{p}<0.05)$. Subjects with normal content in the maxillary sinus present lower ostium height values. There were statistically significant with presence of and higher ostium height values and antral pseudocyst $(\mathrm{p}<0.01)$.

Conclusion: The current study results demonstrated that some sinusopathies were significantly related to dimensions of maxillary sinus drainage.
\end{abstract}

\section{Introduction}

Maxillary sinuses (MS) appear as a small invagination during the fourth month of foetal life, remaining as such and limited to the medial portion of the maxillary bone. With the individual's growth, sinuses expand and occupy a large extension within the maxillary bone, reaching the maximum development after the second dentition (Sperber, 1980). They may vary in terms of shape and size, with variations between the right and left sides not being infrequent in the same individual.

The paranasal sinuses are directly related to the roots of upper teeth, causing them to be affected by inflammatory odontogenic processes which alter their content and normal physiology (Khojastepour, Mirhadi, \& Mesbahi, 2015; Maillet, Bowles, McClanahan, John, \& Ahmad, 2011). In this way, changes of inflammatory origin may lead to symptoms similar to those found in sinusal pathologies, in which the reciprocal is true.
The lining mucosa of the sinuses resembles that of respiratory epithelium as it contains ciliated cylindrical cells and caliciform cells producing mucus. This mucus is normally drained by a system called ostiomeatal complex, which is composed of ostium of maxillary sinus, ethmoidal infundibulum, uncinate process, ethmoidal bulla, semilunar hiatus, frontal recess, middle meatus and middle conchae (Kinsui, Guilherme, \& Yamashita, 2002). After reaching the drainage opening of the maxillary sinus (i.e. ostium), the mucus passes through a small canal which communicates with the nasal cavity (i.e. infundibulum). Both are medially limited by the uncinate process and laterally by the ethmoidal bulla. In turn, the infundibulum opens near into the middle nasal conchae called semilunar hiatus (Parks, 2014; Poleti et al., 2014). These drainage structures of maxillary sinuses are anatomically located above their respective floor, which makes drainage dependent basically on the functioning of the ciliary system of the lining epithelium in the inferior-superior direction.

In dentistry, computed tomography allows the practitioner to

\footnotetext{
* Corresponding author at: Department of Orthodontics and Radiology, School of Dentistry, University City of Sao Paulo, Rua Cesario Galeno, 448, Sao Paulo, SP, 03071-000, Brazil.

E-mail address: alfcosta@gmail.com (A.L. Ferreira Costa).
} 
identify and delineate pathological processes in the paranasal sinuses (Bulbul, Yanik, \& Demirpolat, 2017). Cone beam computed tomography (CBCT), a method improved in the late 1990s, has been used to study dentomaxillofacial structures with a significantly lower dose of radiation compared to the fan-beam computed tomography (Scarfe, Farman, \& Sukovic, 2006). This imaging examination method provides high definition images of calcified tissues as well as slices in anatomical planes without overlapping and volumetric aspects of the craniofacial structures (Raghav, Karjodkar, Sontakke, \& Sansare, 2014).

In the literature, one can observe some studies (Da Silva et al., 2017; Maillet et al., 2011; Parks, 2014; Poleti et al., 2014; Raghav et al., 2014) using this examination method for investigation of the physiology and pathologies related to paranasal sinuses, including variation in the ostiomeatal complex. However, there is no study relating the ostial height of maxillary sinus to the presence of sinusopathies of inflammatory origin or length of the infundibulum. These factors may cause the patients to develop a predisposition to inflammatory processes, thus influencing dental semiology as well as planning of surgical interventions for maxillary sinuses, such as arthroplasties and bone grafts in the region of the maxilla.

The objective of this study was to assess the relationship of positioning and dimensions of the maxillary sinus drainage systems with the presence of pathologies.

\section{Materials \& methods}

\subsection{Study sample}

This study followed the universally accepted standards for research in human beings and was approved by the Institutional Review Board of the UNESP (protocol number 55687116.8.0000.0077).

A total of 300 CBCT images (600 maxillary sinuses) were selected from the examination database of patients from at the Oral \& Maxillofacial Radiology Division, School of Dentistry of São José dos Campos, UNESP. The scans were retrieved from the database in the DICOM (Digital Imaging and Communications in Medicine) format. The images were acquired with the scanner i-CAT Next Generation (Imaging Science International, Hatfield, PA, USA) operating at a FOV (field of view) encompassing the entire middle third of the face $(8.0 \mathrm{~cm}$ height), thus enabling full evaluation of the maxillary sinuses and their respective drainage system with a $0.25-\mathrm{mm}$ voxel size.

Two calibrated dentomaxillofacial radiologists reviewed and discussed the СВСТ scans. All the scans were selected according to the following inclusion/exclusion criteria:

\subsection{Inclusion criteria}

Age above 18 years old for both genders; absence of implants or prior surgeries in the posterior regions of the maxilla; absence of inflammatory processes of endodontic origin (periapical inflammatory lesions) near the floor of the corresponding maxillary sinuses which might cause sinusopathies; absence of floor continuity in the cases of sinusopathies; absence of local bone grafts.

\subsection{Exclusion criteria}

Impaired visualization of details; low sharpness images resulting from patients' movements during scanning, or with artifacts.

The images of the right and left maxillary sinuses were analysed individually based on their contents (Carmeli, Artzi, Kozlovsky, Segev, \& Landsberg, 2011; Ritter et al., 2011), resulting in the following classification (Fig. 1):

a) Normal (I): Hypodensity;

b) Presence of mucous thickening (II): Image of density in the soft parts with linear aspect in association with their walls; c) Presence of antral pseudocyst (III): Image of density in the soft parts with circular aspect in association with their walls;

d) Partial opacification (IV): Image of density in the soft parts resulting in partial opacification, but presenting regions of internal hypodensity;

e) Total opacification (V): Image of density in the soft parts resulting in total opacification.

\subsection{Methodology for image analysis}

The scans selected were imported to Xoran software (Xoran Technologies, Ann Arbor, Michigan, USA) and multiplanar reconstruction images were generated. Adjustments were made in the medial and occlusal planes so that the hard palate on the coronal slices was coincident with the horizontal line exhibited on these slices. All measurements were performed by one dentomaxillofacial radiologist with 12 years of experience in interpreting CBCT.

After such adjustment, the entire extension of the right maxillary sinus (and then the left one) was examined individually in the anteroposterior sense on the coronal window in order to determine the lowest point of the floor. In the slice in which this point was identified, the guiding horizontal line of the axial slice was tangentially positioned at the point of interest, remaining fixed on this level and being shown in this position even when the coronal slice was changed, thus emphasising the lowest point of the maxillary sinus floor. Next, the coronal slice showing all structures of the ostiomeatal complex was selected. In this slice, a vertical line was drawn, with the ruler tool, perpendicular to the guiding horizontal line, leaving the centre of the ostium of the maxillary sinus, thus determining the distance (in millimeters) between the ostium and the lowest point of the corresponding maxillary sinus, ostium height (FOD), Fig. 2.

The length of the infundibulum (LI) of the corresponding maxillary sinus was determined in the slice with better visualisation. The tool ruler was used to measure the distance (in $\mathrm{mm}$ ) between the centre of the ostium and the most upper point of the uncinate process, passing through the infundibulum (Fig. 3).

After a 15-day interval, all the measurements were repeated for analysis of intra-rater reliability.

Data on ostium heights, infundibulum length and type of content corresponding to the two maxillary sinuses of each patient were tabulated. Next, statistical test was applied for assessing a possible relationship between these variables.

\subsection{Statistical analysis}

The agreement between the two evaluation times was verified through scatter plots and the Lin's concordance correlation coefficient. The Student $t$-test was used to compare the genders in relation to the linear parameters and also to compare the maxillary sinuses content in relation to the linear parameters. To compare the genders in relation to the maxillary sinuses content, the Chi-Square test was used. The McNemar test was used to compare the sides in relation to the changes. The confidence level used in the analysis was $5 \%$. All data were statistically analyzed using Minitab 16 (Minitab, Inc., State College, PA, USA).

\section{Results}

Analysis of the scatter plots showed that there was an almost perfect concordance to the intra - examiner for two measurement times for FOD and LI on both sides (i.e. right and left sides) and in both genders (males and females), Lin's concordance correlation coeficiente $>0.999$.

Student's $t$-test was used for comparison between genders and the linear measurements (FOD and LI) made. The results are shown in Table 1. It was found that male individuals had, on average, higher values of FOD on both right and left sides and higher values of LI on the 

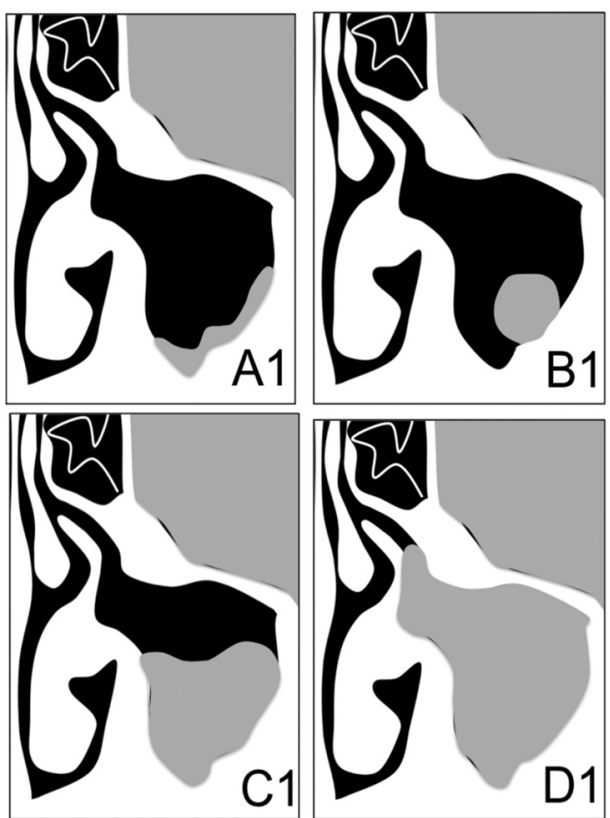
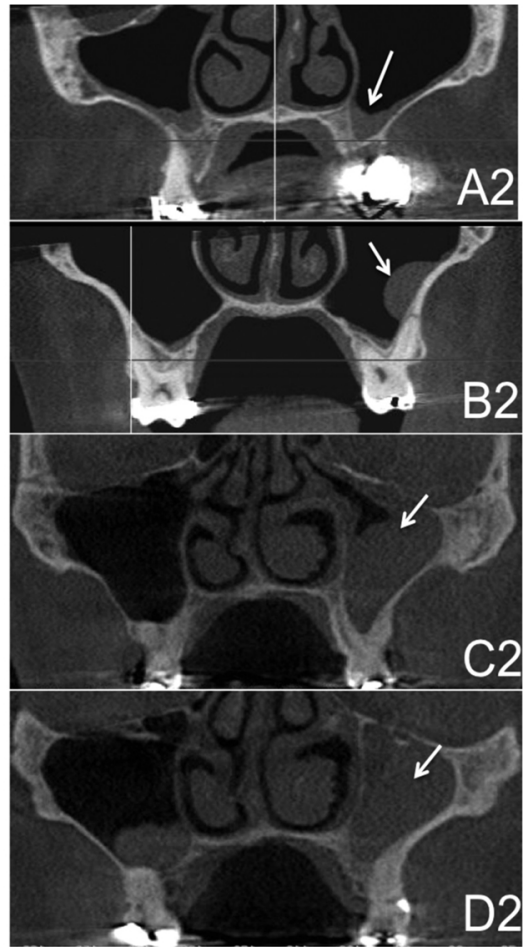

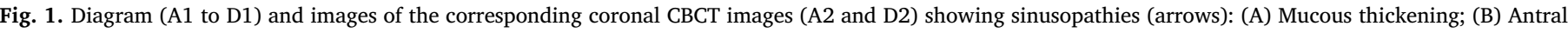
pseudocyst; (C) Partial opacification; and (D) Total opacification.

right side. No statistical differences were found between genders regarding LI on the left side.

Chi-square test was used to compare males and females in relation to the different contents of the maxillary sinus being studied (i.e. I normal aspect, II - mucous thickening, III - presence of antral pseudocyst, IV - partial opacification, and V - total opacification). The results are shown in Table 2.

$P$-values indicate that there were significant differences in the normal aspect (I) and presence of antral pseudocyst (III), with the former being more prevalent in females and the latter more prevalent in males. A significant gender difference was also found for presence of mucous thickening (II) on the left side. In this context, males had a higher percentage of this change than females.

Student's $t$-test was performed to determine the possible relationship between FOD and LI as well as the different contents of the maxillary sinus studied (I to V). As for FOD, a significant difference in the values and presence of type-I content (normal aspect) were observed on both right and left sides. That is, individuals with normal content in the maxillary sinus present lower FOD values. Similarly, a significant difference was found for presence of antral pseudocyst (typeIII content) and higher FOD values. One can hypothesize that higher FOD could affect the sinusal content extravasation, which would change the function of the goblet cells of the sinusal epithelium, altering the function of the ciliary motion and leadind to accumulation of contents in the same cells generating to the formation of antral pseudocyst. As for LI, significant differences in type-III (antral pseudocyst) and type-V (total opacification) contents on the right side were found. Therefore, individuals having presence of antral pseudocyst are more

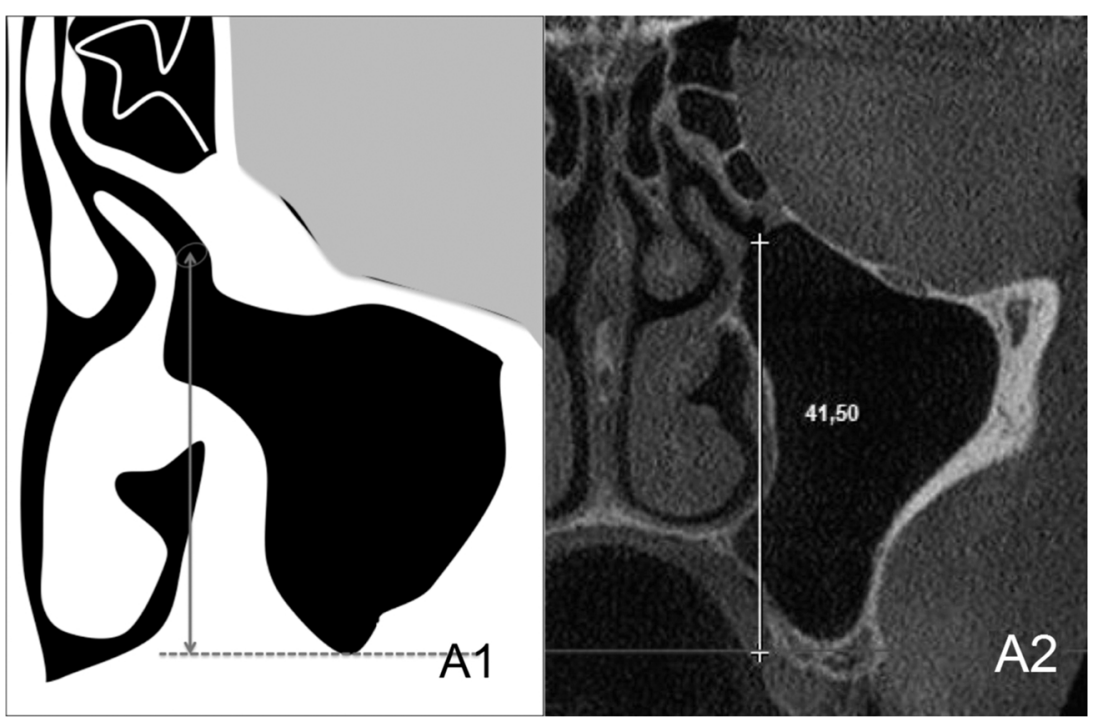

Fig. 2. Diagram (A1) coronal CBCT image (A2) showing height of the maxillary sinus ostium (FOD). 


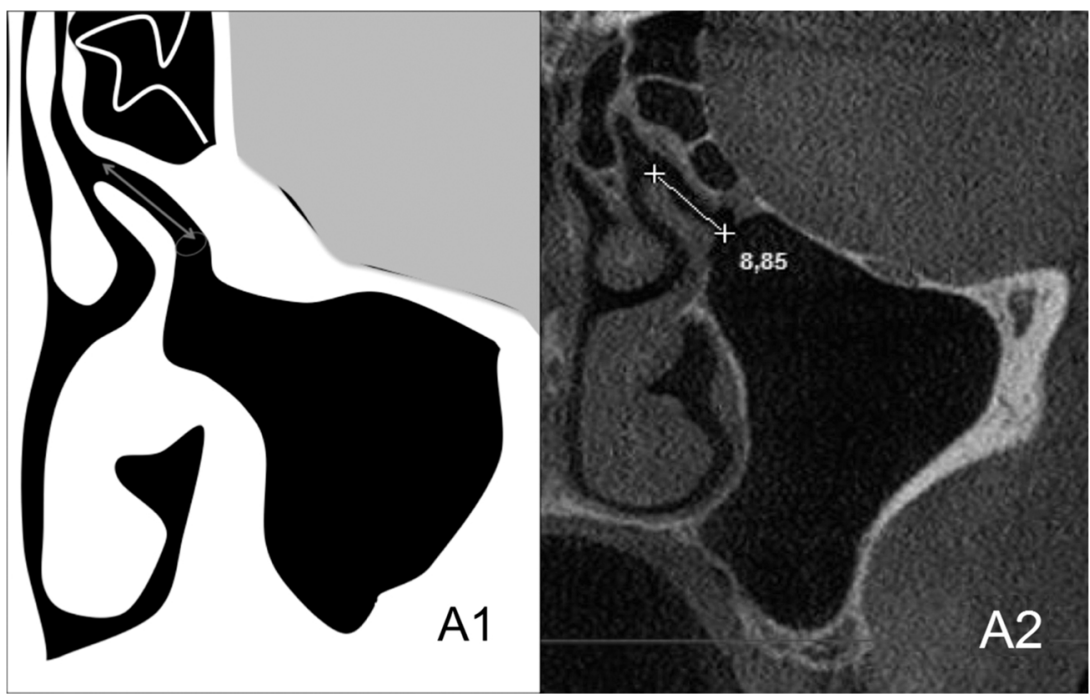

Fig. 3. Diagram (A1) and coronal CBCT image (A2) showing length of the infundibulum of the maxillary sinus (LI).

Table 1

Mean values (DOI and LI) and standard deviation comparing differences between males and females.

\begin{tabular}{lllll}
\hline Variables (mm) & Gender & Mean values & S.D. & $p$-valor \\
\hline FOD_R & F & 31.61 & 4.54 & $0.001^{*}$ \\
FOD_L & M & 34.52 & 5.84 & \\
& F & 31.72 & 4.62 & $0.001^{*}$ \\
LI_R & M & 35.31 & 6.04 & \\
& F & 10.68 & 2.61 & $0.014^{*}$ \\
LI_L & M & 11.49 & 2.77 & \\
& F & 10.46 & 1.97 & 0.135 \\
& M & 10.82 & 2.00 &
\end{tabular}

FOD (height of the ostium); LI (length of the infundibulum); R (right); L (left); Linear measurement (mm); S.D.: standard deviation.

* $p$-value significant at $5 \%$.

Table 2

Distribution of the sample according to contents of the maxillary sinus and gender.

\begin{tabular}{|c|c|c|c|c|c|c|c|}
\hline \multirow[t]{2}{*}{ Side } & \multirow[t]{2}{*}{ Content } & \multirow[t]{2}{*}{ Presence } & \multicolumn{2}{|c|}{ Female } & \multicolumn{2}{|c|}{ Male } & \multirow[t]{2}{*}{$p$-value } \\
\hline & & & $\mathbf{n}$ & $\%$ & $\mathbf{n}$ & $\%$ & \\
\hline \multirow[t]{10}{*}{ Right } & \multirow[t]{2}{*}{ I } & No & 85 & 43.59 & 65 & 61.90 & \multirow[t]{2}{*}{$0.002^{*}$} \\
\hline & & Yes & 110 & 61.90 & 40 & 38.10 & \\
\hline & \multirow[t]{2}{*}{ II } & No & 141 & 72.31 & 67 & 63.81 & \multirow[t]{2}{*}{0.128} \\
\hline & & Yes & 54 & 27.69 & 38 & 36.19 & \\
\hline & \multirow[t]{2}{*}{ III } & No & 187 & 95.90 & 92 & 87.62 & \multirow[t]{2}{*}{$0.007^{*}$} \\
\hline & & Yes & 8 & 4.10 & 13 & 12.38 & \\
\hline & \multirow[t]{2}{*}{ IV } & No & 175 & 89.74 & 93 & 88.57 & \multirow[t]{2}{*}{0.754} \\
\hline & & Yes & 20 & 10.26 & 12 & 11.43 & \\
\hline & \multirow[t]{2}{*}{$\mathrm{V}$} & No & 192 & 98.46 & 103 & 98.10 & \multirow[t]{2}{*}{0.813} \\
\hline & & Yes & 3 & 1.54 & 2 & 1.90 & \\
\hline \multirow[t]{10}{*}{ Left } & \multirow[t]{2}{*}{ I } & No & 87 & 44.62 & 71 & 67.62 & \multirow[t]{2}{*}{$0.001^{*}$} \\
\hline & & Yes & 108 & 55.38 & 34 & 32.38 & \\
\hline & \multirow[t]{2}{*}{ II } & No & 143 & 73.33 & 61 & 58.10 & \multirow[t]{2}{*}{$0.007^{*}$} \\
\hline & & Yes & 52 & 26.67 & 44 & 41.90 & \\
\hline & \multirow[t]{2}{*}{ III } & No & 185 & 94.87 & 89 & 84.76 & \multirow[t]{2}{*}{$0.003^{*}$} \\
\hline & & Yes & 10 & 5.13 & 16 & 15.24 & \\
\hline & \multirow[t]{2}{*}{ IV } & No & 172 & 88.21 & 94 & 89.52 & \multirow[t]{2}{*}{0.731} \\
\hline & & Yes & 23 & 11.79 & 11 & 10.48 & \\
\hline & \multirow[t]{2}{*}{$\mathrm{V}$} & No & 193 & 98.97 & 103 & 98.10 & \multirow[t]{2}{*}{0.527} \\
\hline & & Yes & 2 & 1.03 & 2 & 1.90 & \\
\hline
\end{tabular}

I - normal aspect. II - mucous thickening. III - presence of antral pseudocyst. IV partial opacification. and $\mathrm{V}$ - total opacification.

* $p$-value significant at $5 \%$.
Table 3

Mean values and standard deviation for FOD in right combining the contents of the maxillary sinus.

\begin{tabular}{llllll}
\hline Content & Presence & $\mathrm{N}$ & Mean values & S.D. & $p$-value \\
\hline I & No & 150 & 33.36 & 0.41 & $0.015^{*}$ \\
& Yes & 150 & 31.90 & 0.43 & \\
II & No & 208 & 32.50 & 0.37 & 0.518 \\
& Yes & 92 & 32.91 & 0.50 & \\
III & No & 279 & 32.29 & 0.30 & $0.001^{*}$ \\
& Yes & 21 & 37.10 & 1.13 & \\
IV & No & 268 & 32.68 & 0.32 & 0.579 \\
& Yes & 32 & 32.18 & 0.84 & \\
V & No & 295 & 32.62 & 0.30 & 0.743 \\
& Yes & 5 & 33.35 & 2.07 & \\
& & & & &
\end{tabular}

I - normal aspect. II - mucous thickening. III - presence of antral pseudocyst. IV partial opacification. and V - total opacification.

$\mathrm{N}$ : number; S.D.: standard deviation.

* $p$-value significant at $5 \%$.

likely to have higher LI values, whereas those with total opacification are more likely to have lower LI values (Tables 5 and 6).

McNemar's test was used for analysis of the relationship between the different contents of the maxillary sinus studied (I to V) and the side of their occurrence (i.e. right and left). Table 4 shows frequency distribution and percentage of each change in relation to each side. For type-I content, for instance, 112 individuals $(37.33 \%$ of a total of 300$)$

Table 4

Mean values and standard deviation for FOD in left combining the contents of the maxillary sinus.

\begin{tabular}{llllll}
\hline Content & Presence & $\mathrm{N}$ & Mean values & S.D. & $p$-value \\
\hline I & No & 158 & 33.56 & 0.46 & $0.049^{*}$ \\
& Yes & 142 & 32.33 & 0.42 & \\
II & No & 204 & 32.91 & 0.35 & 0.781 \\
& Yes & 96 & 33.11 & 0.63 & \\
III & No & 274 & 32.66 & 0.33 & $0.001^{*}$ \\
& Yes & 26 & 36.27 & 0.94 & \\
IV & No & 266 & 33.06 & 0.34 & 0.427 \\
& Yes & 34 & 32.32 & 0.87 & \\
V & No & 296 & 32.92 & 0.32 & 0.261 \\
& Yes & 4 & 37.00 & 2.93 &
\end{tabular}

I - normal aspect. II - mucous thickening. III - presence of antral pseudocyst. IV partial opacification. and V - total opacification.

$\mathrm{N}$ : number; S.D.: standard deviation.

* $p$-value significant at $5 \%$. 
Table 5

Mean values and standard deviation for LI in right combining the contents of the maxillary sinus.

\begin{tabular}{llllll}
\hline Content & Presence & $\mathrm{N}$ & Mean values & S.D. & $p$-value \\
\hline I & No & 150 & 11.11 & 0.25 & 0.348 \\
& Yes & 150 & 10.82 & 0.19 & \\
II & No & 208 & 11.02 & 0.19 & 0.616 \\
& Yes & 92 & 10.85 & 0.27 & \\
III & No & 279 & 10.88 & 0.16 & $0.047^{*}$ \\
& Yes & 21 & 12.10 & 0.56 & \\
IV & No & 268 & 10.91 & 0.15 & 0.540 \\
& Yes & 32 & 11.41 & 0.78 & \multirow{2}{*}{$0.031^{*}$} \\
V & No & 295 & 10.98 & 0.16 & \\
& Yes & 5 & 9.84 & 0.35 & \\
\hline
\end{tabular}

I - normal aspect. II - mucous thickening. III - presence of antral pseudocyst. IV partial opacification. and V - total opacification.

$\mathrm{N}$ : number; S.D.: standard deviation.

* $p$-value significant at $5 \%$.

Table 6

Mean values and standard deviation for LI in left combining the contents of the maxillary sinus.

\begin{tabular}{llllll}
\hline Content & Presence & $\mathrm{N}$ & Mean & D.P. & $p$-value \\
\hline I & No & 158 & 10.51 & 0.16 & 0.504 \\
& Yes & 142 & 10.66 & 0.17 & \\
II & No & 204 & 10.56 & 0.14 & 0.794 \\
& Yes & 96 & 10.63 & 0.21 & \\
III & No & 274 & 10.58 & 0.12 & 0.979 \\
& Yes & 26 & 10.57 & 0.41 & \\
IV & No & 266 & 10.64 & 0.12 & 0.120 \\
& Yes & 34 & 10.15 & 0.29 & \\
V & No & 296 & 10.59 & 0.12 & 0.495 \\
& Yes & 4 & 10.15 & 0.56 & \\
& & & & &
\end{tabular}

I - normal aspect. II - mucous thickening. III - presence of antral pseudocyst. IV partial opacification. and V - total opacification. " $p$-value significant at $5 \%$. $\mathrm{N}$ : number; S.D.: standard deviation.

\section{Percentage of agreement between right and left sides}$$
100
$$

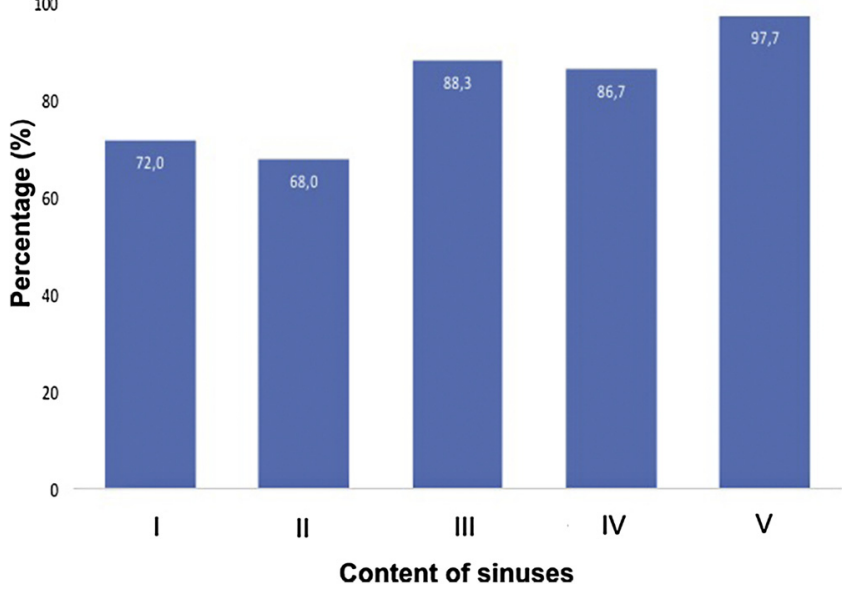

Fig. 4. Percentage of agreement between right and left sides. and contents of the maxillary sinuses; I - normal aspect. II - mucous thickening. III - presence of antral pseudocyst. IV - partial opacification. and V - total opacification.

did not exhibit this change on none of the sides and 104 individuals (34.70\% of a total of 300 ) had it on both sides. Therefore, the concordance percentage between the sides was $72.0 \%$, as shown in Fig. 4 . No significant differences were found between the sides regarding any changes $(\mathrm{p}>0.05)$

\section{Discussion}

Maxillary sinuses are the largest of the paranasal sinuses and whose drainage is performed through the ostiomeatal complex, which comprises the ostium and infundibulum, towards the middle meatus. These structures are at an upper position in relation to the lower limit of the maxillary sinus (i.e. floor), thus making the contents (e.g. mucous secretions from caliciform cells of the lining epithelium, by-products from sinusopathies) present in the maxillary antrum be gradually transported through the ciliary beating of sinusal lining cells in order to overcome the gravitational force (Block \& Dastoury, 2014; Da Silva et al., 2017).

In this way, the present study has investigated how two factors (distance from the most inferior point of the maxillary sinus floor to the drainage ostium and also length of infundibulum) might influence the type of sinusal content (i.e. normoaerated or not) by using CBCT, which is a gold-standard modality in the study of paranasal sinuses (Block \& Dastoury, 2014; Dobele, Kise, Apse, Kragis, \& Bigestans, 2013).

In our results, it was observed that higher values of FOD and LI were found in male individuals (Table 1). This finding is corroborated by the fact that men have a higher physical height, which reflects into the dimension of sinusal structures.

Our findings also indicate that the normal aspect (type-I content) was more prevalent in female individuals, which was corroborated by the lower values of FOD and LI found among women, suggesting that the shorter the ostium height and infundibulum, the easier the drainage of sinusal content, thus reducing the chance of accumulation of secretion. In male individuals, we have observed a significant relationship between presence of mucous thickening and presence of antral pseudocyst (Table 2), a finding corroborating the fact that men have larger drainage structures. Anatomically, this could make it difficult to empty the content from the maxillary sinus, resulting in its opacification by either mucous thickening or formation of antral pseudocyst.

Lower FOD values indicated a greater relationship with normal sinusal content, whereas higher FOD values indicated presence of mucous thickening (Tables 1 and 2), which once again reinforces the influence of ostium height on facilitating the sinus drainage (Dobele et al., 2013).

On the other hand, LI values (Tables 3 and 4 ) indicated that there was an inverse relationship with the presence of total opacification as well as a direct relationship with the presence of antral pseudocyst. This might lead us to conclude that, differently from FOD, LI is not a determining variable for a satisfactory drainage process in the maxillary sinuses or even when there is an intense accumulation of sinusal content - for instance, represented by high FOD values and total opacification. The mean LI is not a determining factor for drainage of the sinusal content, meaning that there might be already an obstruction in the ostiomeatal complex, for instance. On the other hand, the presence of antral pseudocyst may be associated with a problem affecting not only the drainage, but also intrinsic structures of the caliciform cells, leading to their obstruction with accumulation of contents, but with no relationship with any possible anatomical difficult (Cornet, Georgalas, Reinartz, \& Fokkens, 2013; Da Silva et al., 2017; Dobele et al., 2013).

It was also observed that there was no relationship between distribution of content types and sides (i.e. right and left). This is understandable as the anatomical distances in general follow a pattern in the same individual, unless there is a previous surgical intervention on one of the sides or any discrepancy due to asymmetric growth processes, but cases like this were excluded from our sample.

This study has some limitations that cannot be underestimated. Firstly, the CBCTs performed in our study have been required for diagnosis of sinuses. Severity and spectrum of the illnesses may differ from other institutions, and this should be taken into account when generalizing our results. Secondly, ethnicity can be one of the factors that may account for the differences in the distribution of variations. Therefore, the characteristics of the selected subjects should be considered when comparing our conclusions 


\section{Conclusion}

Lower values of ostium height and length of the infundibulum are associated with female gender, whereas higher values are associated with male gender. Ostium height was shown to be a determining factor for the presence of normal content in the maxillary sinus, being inversely proportional to it. Length of the infundibulum was not found to be a factor influencing the normal aspect of sinusal content, rather than total opacification and presence of antral pseudocyst.

\section{Competing interests}

The authors declare that they have no competing interests.

\section{References}

Block, M. S., \& Dastoury, K. (2014). Prevalence of sinus membrane thickening and association with unhealthy teeth: A retrospective review of 831 consecutive patients with 1,662 cone-beam scans. Journal of Oral and Maxillofacial Surgery, 72(12), 2454-2460.

Bulbul, E., Yanik, B., \& Demirpolat, G. (2017). Detection of dental pathologies in routine paranasal CT scans: A retrospective study. Journal of Clinical and Diagnostic Research, 11(7), TC17-TC20.

Carmeli, G., Artzi, Z., Kozlovsky, A., Segev, Y., \& Landsberg, R. (2011). Antral computerized tomography pre-operative evaluation: Relationship between mucosal thickening and maxillary sinus function. Clinical Oral Implants Research, 22(1), 78-82.

Cornet, M. E., Georgalas, C., Reinartz, S. M., \& Fokkens, W. J. (2013). Long-term results of functional endoscopic sinus surgery in children with chronic rhinosinusitis with nasal polyps. Rhinology, 51(4), 328-334.
Da Silva, A. F., Froes, G. R., Jr, Takeshita, W. M., Da Fonte, J. B., De Melo, M. F., \& Sousa Melo, S. L. (2017). Prevalence of pathologic findings in the floor of the maxillary sinuses on cone beam computed tomography images. European Journal of General Dentistry, 65(2), 28-32.

Dobele, I., Kise, L., Apse, P., Kragis, G., \& Bigestans, A. (2013). Radiographic assessment of findings in the maxillary sinus using cone-beam computed tomography. Stomatologija, 15(4), 119-122.

Khojastepour, L., Mirhadi, S., \& Mesbahi, S. A. (2015). Anatomical variations of ostiomeatal complex in CBCT of patients seeking rhinoplasty. Journal of Dentistry (Shiraz), 16(1), 42-48.

Kinsui, M. M., Guilherme, A., \& Yamashita, H. K. (2002). Variações anatômicas e sinusopatias: Estudo por tomografia computadorizada. Revista Brasileira de Otorrinolaringologia, 68, 645-652.

Maillet, M., Bowles, W. R., McClanahan, S. L., John, M. T., \& Ahmad, M. (2011). Conebeam computed tomography evaluation of maxillary sinusitis. Journal of Endodontics, 37(6), 753-757.

Parks, E. T. (2014). Cone beam computed tomography for the nasal cavity and paranasal sinuses. Dental Clinics of North America, 58(3), 627-651.

Poleti, M. L., Paes da Silva Ramos Fernandes, L. M., Oliveira-Santos, C., Capelozza, A. L. Chinellato, L. E., \& Rubira-Bullen, I. R. (2014). Anatomical variation of the maxillary sinus in cone beam computed tomography. Case Reports in Dentistry, 2014, 707261.

Raghav, M., Karjodkar, F. R., Sontakke, S., \& Sansare, K. (2014). Prevalence of incidental maxillary sinus pathologies in dental patients on cone-beam computed tomographic images. Contemporary Clinical Dentistry, 5(3), 361-365.

Ritter, L., Lutz, J., Neugebauer, J., Scheer, M., Dreiseidler, T., Zinser, M. J., et al. (2011). Prevalence of pathologic findings in the maxillary sinus in cone-beam computerized tomography. Oral Surgery, Oral Medicine, Oral Pathology, Oral Radiology, and Endodontology, 111(5), 634-640.

Scarfe, W. C., Farman, A. G., \& Sukovic, P. (2006). Clinical applications of cone-beam computed tomography in dental practice. Journal of the Canadian Dental Association, 72(1), 75-80.

Sperber, G. H. (1980). Applied anatomy of the maxillary sinus. Journal of the Canadian Dental Association, 46(6), 381-386. 